PROCEEDINGS OF THE

AMERICAN MATHEMATICAL SOCIETY

Volume 135, Number 12, December 2007, Pages 3783-3788

S 0002-9939(07)08948-4

Article electronically published on September 12, 2007

\title{
EXAMPLES OF FANO VARIETIES OF INDEX ONE THAT ARE NOT BIRATIONALLY RIGID
}

\author{
ANA-MARIA CASTRAVET
}

(Communicated by Ted Chinburg)

\begin{abstract}
A conjecture of Pukhlikov states that a smooth Fano variety of dimension at least 4 and index one is birationally rigid. We show that a general member of the linear system given by the ample generator of the Picard group of the moduli space of stable, rank 2 bundles with fixed determinant of odd degree on a curve of genus at least 3 is not birationally rigid.
\end{abstract}

\section{Pukhlikov's conjecture}

The following is a conjecture of Pukhlikov:

Conjecture 1.1 ([י 4 , Conjecture 5.1]). Let $V$ be a smooth Fano variety of dimension $\operatorname{dim} V \geq 4$ with Picard group Pic $(V)$ generated by the canonical class $K_{V}$. Then $V$ is birationally rigid. If $\operatorname{dim} V \geq 5$, then $V$ is superrigid.

Conjecture 1.1 was proved for a large class of Fano complete intersections [P1], P3, P4, dF, dFEM. More examples are given by complete intersections in weighted projective spaces $[\mathrm{P} 2$. Conjecture 1.1 is a generalization of the famous theorem of Iskovskikh and Manin [IM] that states that a smooth quartic threefold is birationally rigid, and therefore not rational.

In this note we give counterexamples (in arbitrarily large dimension) to Conjecture 1.1 using moduli spaces of bundles on curves. I am grateful to Professor János Kollár for telling me about this question and suggesting that I look at sections of the theta divisor on moduli spaces of bundles on curves. I thank Jenia Tevelev and Sean Keel for reading this note and for helpful suggestions.

We recall the basic definitions about birational rigidity from [P4]. Let $V$ be a uniruled $\mathbb{Q}$-Gorenstein variety with terminal singularities. For an effective divisor $D \neq 0$ on $V$, one defines the canonical threshold of canonical adjunction $c(D)$ of the divisor $D$ as follows:

$$
c(D)=\sup \left\{\quad b / a \quad\left|\quad b, a \in \mathbb{Z}_{+} \backslash\{0\}, \quad\right| a D+b K_{X} \mid \neq \emptyset\right\} .
$$

(If there are no $a, b \in \mathbb{Z}_{+} \backslash\{0\}$ such that $\left|a D+b K_{X}\right| \neq \emptyset$, then we set $c(D)=0$.)

Note, for an effective divisor $D \neq 0$ on a Fano variety $V$ with $\operatorname{Pic}(V)=\mathbb{Z} K_{V}$, the canonical threshold $c(D)$ is the number $-m$, if $D=m K_{V}$ in $\operatorname{Pic}(V)$. Clearly, in this case $c(D)>0$.

Received by the editors May 5, 2006 and, in revised form, September 17, 2006.

2000 Mathematics Subject Classification. Primary 14E07; Secondary 14H60.

(C)2007 American Mathematical Society

Reverts to public domain 28 years from publication 
In what follows all varieties are assumed to be $\mathbb{Q}$-factorial with terminal singularities. For simplicity, we work over an algebraically closed field of characteristic zero.

Definition 1.2 ([P4, Def. 5.1]). A variety $V$ is called birationally rigid, if for any $V^{\prime}$, any birational map $\phi: V \rightarrow V^{\prime}$ and any moving linear system $\Sigma^{\prime}$ on $V^{\prime}$, there exists a birational self-map $\alpha: V \rightarrow V$ such that if $\Sigma$ is the birational transform of $\Sigma^{\prime}$ via the composition $\phi \circ \alpha$ (i.e., the linear system induced on $V$ when composing with $\phi \circ \alpha)$, one has $c(\Sigma) \leq c\left(\Sigma^{\prime}\right)$. The variety $V$ is called birationally superrigid, if one may always take $\alpha=i d$.

The following proposition is an immediate consequence of the above definitions:

Proposition 1.3 ([P4, Prop. 5.1]). Let $V$ be a smooth Fano variety with Pic $(V)=$ $\mathbb{Z} K_{V}$. If $V$ is birationally rigid, then it is impossible to have a birational map $V \rightarrow V^{\prime}$, with $V^{\prime} \rightarrow S^{\prime}$ a morphism with uniruled general fiber and $S^{\prime}$ a projective variety of dimension $\operatorname{dim} S^{\prime} \geq 1$.

Proof. Assume there is a birational map $V \rightarrow V^{\prime}$, with $\pi: V^{\prime} \rightarrow S^{\prime}$ a morphism with uniruled general fiber and $\operatorname{dim} S^{\prime} \geq 1$. Let $D^{\prime}$ be an effective Cartier divisor on $S^{\prime}$ and let $\Sigma^{\prime}=\pi^{*} D^{\prime}$. By Definition 1.2, there is a birational map $\alpha: V \rightarrow V$ such that if $\Sigma$ is the birational transform of $\Sigma^{\prime}$ via the composition $\phi \circ \alpha$, then $c(\Sigma) \leq c\left(\Sigma^{\prime}\right)$. Note that since $V$ is a Fano variety with $\operatorname{Pic}(V)=\mathbb{Z} K_{V}$, one has $c(\Sigma)>0$.

We claim that $c\left(\Sigma^{\prime}\right)=0$ : if there are $a, b>0$ such that $a \pi^{*} D^{\prime}+b K_{V^{\prime}}$ is effective on $V^{\prime}$, then for a general fiber $F$ of $\pi$ (choose $F$ uniruled, in the smooth locus of $\pi$ and such that it is not contained in the divisor $\left.a \pi^{*} D^{\prime}+b K_{V^{\prime}}\right)$, one has that the divisor

$$
\left(a \pi^{*} D^{\prime}+b K_{V^{\prime}}\right)_{\mid F}=\left(b K_{V^{\prime}}\right)_{\mid F}=b K_{F}
$$

is effective on $F$. This is a contradiction, since $F$ uniruled implies that $\mathrm{H}^{0}\left(b K_{F}\right)=0$ for all $b>0[\mathrm{~K}]$.

Note that since the condition of being uniruled is a closed condition $[\mathrm{K}$, one may drop the word "general" from the statement of Proposition 1.3. Moreover, since the condition of being uniruled is a birational property, one may as well replace the condition of having a morphism $V^{\prime} \rightarrow S^{\prime}$ with having a birational map $V^{\prime} \rightarrow S^{\prime}$ with the same properties.

To geometrically motivate Definition [1.2, we recall the other definition of birational rigidity from $\mathrm{Co}$. Recall that a morphism $f: V \rightarrow S$ is called a Mori fiber space if it has relative Picard number $1,-K_{V}$ is relatively ample for $f$ and $\operatorname{dim} S<\operatorname{dim} V$. Note, Fano varieties with Picard number 1 are trivially Mori fiber spaces. A birational map $\phi: V \rightarrow V^{\prime}$ to another Mori fiber space $f^{\prime}: V^{\prime} \rightarrow S^{\prime}$ is square if there is a birational map $h: S \rightarrow S^{\prime}$ such that $h \circ f=f^{\prime} \circ \phi$ and the map $\phi$ induces an isomorphism on the general fibers.

Definition 1.4 ([Co, Def. 1.3]). A Mori fiber space $f: V \rightarrow S$ is called birationally rigid if for any birational map $\phi: V \rightarrow V^{\prime}$ to another Mori fiber space $f^{\prime}: V^{\prime} \rightarrow S^{\prime}$, there is a birational self-map $\alpha: V \rightarrow V$ such that $\phi \circ \alpha$ is square. If for any $\phi$ as above it follows that $\phi$ is square, then $V$ is called birationally superrigid.

A Mori fiber space that satisfies Definition 1.2 also satisfies Definition 1.4 (by the Sarkisov program, based on the Noether-Fano-Iskovskikh inequalities; this is known 
in dimension 3 and conjectured in higher dimensions) . By the Mori program, any uniruled variety is birational to a Mori fiber space; hence, if in addition one assumes the Mori program, the two definitions are equivalent.

It follows from Definition 1.4 that if $V$ is a smooth Fano variety with Picard number 1 and birationally rigid, then there is no birational map $\phi: V \rightarrow V^{\prime}$, with $f^{\prime}: V^{\prime} \rightarrow S^{\prime}$ another Mori fiber space with $\operatorname{dim} S^{\prime}>0$. From the Mori program for the relative case, one deduces Proposition 1.3. In particular, note that if $V$ is a smooth Fano variety with Picard number 1 and birationally rigid, then for any $V^{\prime}$ another Fano variety of Picard number 1, if $V^{\prime}$ is birational to $V$, then $V \cong V^{\prime}$.

\section{Counterexamples using moduli spaCes of BundLes on CURVES}

Let $C$ be a smooth projective curve over $\mathbb{C}$ of genus $g \geq 3$. Fix $\xi$ to be a degree 1 line bundle on $C$. Let $M$ be the moduli space of stable, rank 2 vector bundles on $C$ with determinant $\xi$. The moduli space $M$ is a smooth, projective, variety of dimension $3 g-3$. The Picard group of $M$ is $\mathbb{Z}[\mathrm{DN}]$. Let $\Theta$ be the ample generator. In fact, $\Theta$ is very ample $\mathrm{BV}$. Then $K_{M} \cong-2 \Theta[\mathrm{R}$.

Let $N$ be a non-singular element of the linear system $|\Theta|$. Let $\Theta^{\prime} \in \operatorname{Pic}(N)$ be the restriction of $\Theta$ to $N$. The canonical bundle of $N$ is $-\Theta^{\prime}$. Since $g \geq 3$, by Lefschetz's theorem, $\Theta^{\prime}$ generates $\operatorname{Pic}(N)$. Therefore, the variety $N$ satisfies the conditions in Conjecture 1.1. We prove the following:

Proposition 2.1. If $N$ is a general element of the linear system $|\Theta|$, then $N$ is a smooth Fano variety with $\operatorname{Pic}(N) \cong \mathbb{Z} K_{N}$ that is not birationally rigid.

Proof. We start with a general construction. Let $e \geq 0$ and let $\mathcal{L}$ be a line bundle of degree $-e$ on $C$. Denote by $V_{\mathcal{L}}$ the space of extensions $\operatorname{Ext}^{1}\left(\mathcal{L}^{-1} \otimes \xi, \mathcal{L}\right) \cong$ $\mathrm{H}^{1}\left(C, \mathcal{L}^{2} \otimes \xi^{-1}\right)$. Then $V_{\mathcal{L}}$ parametrizes extensions of the form

$$
0 \rightarrow \mathcal{L} \rightarrow \mathcal{E} \rightarrow \mathcal{L}^{-1} \otimes \xi \rightarrow 0
$$

The dimension of $V_{\mathcal{L}}$ is $2 e+g$. Clearly, any two non-zero elements in $V_{\mathcal{L}}$ which differ by a scalar define isomorphic vector bundles $\mathcal{E}$. Therefore, the isomorphism classes of non-trivial extensions as above are parametrized by the projective space $\mathbb{P}\left(V_{\mathcal{L}}\right)$. The locus $Z_{\mathcal{L}} \subset \mathbb{P}\left(V_{\mathcal{L}}\right)$ of extensions (因) with $\mathcal{E}$ unstable is an irreducible subvariety of codimension at least $g$ [Ca, Lemma 2.1]. There is a well-defined morphism

$$
\kappa_{\mathcal{L}}: \mathbb{P}\left(V_{\mathcal{L}}\right) \backslash Z_{\mathcal{L}} \rightarrow M
$$

that associates to an extension (函) the isomorphism class of $\mathcal{E}$. By $\mathrm{Ca}, 2.3(1)$, or Lemma A.1]

$$
\kappa_{\mathcal{L}}^{*} \Theta \cong \mathcal{O}(2 e+1)
$$

Consider the case when $e=g-1$. Then $\mathbb{P}\left(V_{\mathcal{L}}\right) \cong \mathbb{P}^{3 g-3}$ and $\kappa_{\mathcal{L}}^{*} \Theta \cong \mathcal{O}(2 g-1)$. The following claim is a standard fact. For convenience, we include a short proof.

Claim 2.2. If $\mathcal{L}$ is a line bundle of degree $1-g$, the morphism $\kappa_{\mathcal{L}}$ in (2.1) is birational.

Proof of Claim 2.2, Note that for any $\mathcal{E} \in M$, by Riemann-Roch one has

$$
\chi\left(\mathcal{E}^{*} \otimes \mathcal{L}^{-1} \otimes \xi\right)=1
$$

Hence, for any $\mathcal{E} \in M$ one has

$$
\operatorname{Hom}\left(\mathcal{E}, \mathcal{L}^{-1} \otimes \xi\right) \cong \mathrm{H}^{0}\left(\mathcal{E}^{*} \otimes \mathcal{L}^{-1} \otimes \xi\right) \neq 0 .
$$


If for general $\mathcal{E} \in M$, any non-zero morphism $\phi: \mathcal{E} \rightarrow \mathcal{L}^{-1} \otimes \xi$ is surjective, then we are done, as $\phi$ determines uniquely (up to scaling) the extension (図), and by dimension considerations, one must have that

$$
\mathrm{h}^{0}\left(\mathcal{E}^{*} \otimes \mathcal{L}^{-1} \otimes \xi\right)=1
$$

(i.e., the fiber of $\kappa$ at a general point contains a unique closed point).

We prove that for general $\mathcal{E}$, a non-zero morphism $\phi: \mathcal{E} \rightarrow \mathcal{L}^{-1} \otimes \xi$ must be surjective. Note that if $\phi$ is not surjective, then its image is $\mathcal{L}^{-1} \otimes \xi(-D)$, for some effective divisor $D$ of degree $d>0$, and there is an exact sequence:

$$
0 \rightarrow \mathcal{L}(D) \rightarrow \mathcal{E} \rightarrow \mathcal{L}^{-1} \otimes \xi(-D) \rightarrow 0 .
$$

It follows from the stability of $\mathcal{E}$ that $d<g$. For each $0<d<g$ construct the total space $\mathbb{P}_{d}$ of extensions (2.2) by letting $D$ vary in $\operatorname{Sym}^{d}(C)$ : the space $\mathbb{P}_{d}$ is a projective bundle over $\operatorname{Sym}^{d}(C)$ with fiber at $D$ isomorphic to $\mathbb{P}\left(V_{\mathcal{L}(D)}\right)$. The dimension is:

$$
\operatorname{dim} \mathbb{P}_{d}=d+\operatorname{dim} V_{\mathcal{L}(D)}-1=d+2(g-1-d)+g-1=3 g-3-d .
$$

The vector bundle which is the middle term of the universal extension over $\mathbb{P}_{d}$ induces a rational map $\rho: \mathbb{P}_{d} \rightarrow M$. Since $d>0$, the map $\rho$ is not dominant. Therefore, a general $\mathcal{E} \in M$ will not sit in an exact sequence (2.2).

From the previous discussion and since $\Theta$ is very ample, one has the following:

Claim 2.3. If $N$ is a general element of the linear system $|\Theta|$, then $N$ is birational to an irreducible hypersurface $X_{2 g-1}$ in $\mathbb{P}^{3 g-3}$ of degree $2 g-1$.

Proposition 2.1 follows now from Claim 2.3 and Lemma 2.4

Lemma 2.4. For any irreducible hypersurface $X_{d} \subset \mathbb{P}^{n}$ of degree $d<n$ (possibly singular) there is a rational map $\rho: X \rightarrow S, \operatorname{dim} S>0$, with uniruled fibers.

Proof. Let $S$ be a general pencil of hyperplanes $\left\{H_{s}\right\}_{s \in S}$ in $\mathbb{P}^{n}$. Then $X_{s}=X \cap H_{s}$ are hypersurfaces of degree $d$ in $H_{s} \cong \mathbb{P}^{n-1}$. A smooth hypersurface of degree $d<n$ in $\mathbb{P}^{n-1}$ is Fano; hence, it is rationally connected $\mathrm{KMM}$. It follows by deformation theory that any irreducible hypersurface of degree $d<n$ in $\mathbb{P}^{n-1}$ is uniruled. Hence, for all $s \in S$ such that $X_{s}$ is irreducible, $X_{s}$ is uniruled. Therefore, the induced rational map $\rho: X \rightarrow S$ has uniruled fibers.

Remark 2.5. The cohomology group $\mathrm{H}^{4}(M ; \mathbb{Q})$ has two independent generators $\mathbb{N}$. Hence, by Lefschetz's theorem, if $g \geq 4$, the rank of $H^{4}(N ; \mathbb{Q})$ is also 2. Since again by Lefschetz's theorem, the cohomology group $\mathrm{H}^{4}$ of any smooth complete intersection of dimension $\geq 5$ is of rank 1 , it follows that $N$ is not a complete intersection.

\section{Description of the hypersurface $X_{2 g-1} \subset \mathbb{P}^{3 g-3}$}

Our construction of the map $\kappa_{\mathcal{L}}$ is a variant of the construction of Bertram [B]. The above results about the map $\kappa_{\mathcal{L}}(\operatorname{deg} \mathcal{L}=1-g)$ also follow from $[\mathrm{B}$. We chose to include the above considerations (which are enough for the purpose of this note) because of their simplicity and to avoid referring to the technical results in B. However, Bertram's powerful construction gives a precise description of the hypersurface $X_{2 g-1} \subset \mathbb{P}^{3 g-3}$. We describe this below. 
Let $\mathcal{L}$ be a line bundle of degree $1-g$ on $C$. Then $C$ has a natural embedding $C \subset \mathbb{P}\left(V_{\mathcal{L}}\right) \cong \mathbb{P}^{3 g-3}$ given by $\mathcal{L}^{-2} \otimes \xi \otimes K_{C}$, since by Serre duality one has:

$$
V_{\mathcal{L}} \cong \mathrm{H}^{1}\left(C, \mathcal{L}^{2} \otimes \xi^{-1}\right) \cong \mathrm{H}^{0}\left(C, \mathcal{L}^{-2} \otimes \xi \otimes K_{C}\right)^{*}
$$

Let $\operatorname{Sec}^{k}(C)$ be the $(k+1)$-secant variety of $C$ (i.e., the closure in $\mathbb{P}^{3 g-3}$ of the union of all the $k$-planes spanned by $k+1$ distinct points on $C$ ).

Theorem 3.1 ([B], Thm. 1]). There is a sequence of blow-ups $\pi: \tilde{\mathbb{P}}_{\mathcal{L}} \rightarrow \mathbb{P}\left(V_{\mathcal{L}}\right)$ with smooth centers (starting with the blow-up of $\mathbb{P}\left(V_{\mathcal{L}}\right)$ along $C$ ) that resolves the rational map $\kappa_{\mathcal{L}}: \mathbb{P}\left(V_{\mathcal{L}}\right) \rightarrow M$ into a morphism $\tilde{\mathbb{P}}_{\mathcal{L}} \rightarrow M$. There are $g$ exceptional divisors $E_{0}, E_{1}, \ldots, E_{g-1}$, and $E_{k}$ dominates the secant variety $\operatorname{Sec}^{k}(C)$ for every $k$.

Theorem 3.2 ([B], Thm. 2, Prop. 4.7]). There is a natural identification

$$
H^{0}(M, \Theta) \cong H^{0}\left(\tilde{\mathbb{P}}_{\mathcal{L}},(2 g-1) H-(2 g-3) E_{0}-(2 g-5) E_{1}-\ldots-E_{g-2}\right) .
$$

It follows from Theorem 3.2 that the proper transform $\tilde{X}$ in $\tilde{\mathbb{P}}_{\mathcal{L}}$ of the hypersurface $X_{2 g-1} \subset \mathbb{P}^{3 g-3}$ of Claim 2.3 is a general member of the linear system in (3.1). Hence, by Bertini, $\tilde{X}$ is smooth.

Proposition 3.3. The singular locus of the hypersurface $X_{2 g-1} \subset \mathbb{P}^{3 g-3}$ has codimension $\geq g-2$. Hence, if $g \geq 4$, then $X$ is normal and the canonical bundle $K_{X}$ is Cartier. Moreover, in this case $X$ has terminal singularities.

Proof. Since the proper transform $\tilde{X}$ of $X$ is smooth, it follows that $X$ is smooth outside $\operatorname{Sec}^{g-1}(C) \cap X$. Since a general $X$ does not contain $\operatorname{Sec}^{g-1}(C)$, it follows that the singular locus of $X$ has dimension at most $\operatorname{dim} \operatorname{Sec}^{g-1}(C)-1=2 g-2$. It is well-known that hypersurfaces $X$ whose singular locus has codimension at least 2 are normal and the canonical class $K_{X}$ is Cartier. Hence, if $g \geq 4$, then $X$ is normal and has the canonical class:

$$
K_{X}=\mathcal{O}_{X}(1-g) .
$$

Consider the resolution $\pi: \tilde{X} \rightarrow X$. The canonical class of $\tilde{\mathbb{P}}_{\mathcal{L}}$ is given by:

$$
K_{\tilde{\mathbb{P}}_{\mathcal{L}}}=-(3 g-2) H+(3 g-5) E_{0}+(3 g-7) E_{1}+\ldots+(g-1) E_{g-2} .
$$

The canonical class of $\tilde{X}$ is given by:

$$
K_{\tilde{X}}=\left(K_{\tilde{\mathbb{P}}_{\mathcal{L}}}+\tilde{X}\right)_{\mid \tilde{X}}=-(g-1) H+(g-2) E_{0}+(g-2) E_{1}+\ldots+(g-2) E_{g-2} .
$$

Hence, $X$ has terminal singularities.

\section{REFERENCES}

[B] Bertram, A., Moduli of rank 2 vector bundles, theta divisors, and the geometry of curves in projective space, J. Differential Geometry, 35, (1992), 429-469 MR.1158344 (93g:14037)

[BV] Brivio, S., Verra, A., The theta divisor of $\operatorname{SU}_{C}(2,2 d)^{s}$ is very ample if $C$ is not hyperelliptic, Duke Math. J., 82, (1996), No. 3, 503-552 MR.1387683(97e:14017)

[Ca] Castravet, A.-M., Rational families of vector bundles on curves, International Journal of Mathematics, 15, No. 1 (2004); arxiv:math. AG/0302133 and math. AG/0302135 MR2039210 (2005i:14038)

[Co] Corti, A., Singularities of linear systems and 3-fold birational geometry, in Explicit birational geometry of 3-folds, 259-312, Cambridge Univ. Press, Cambridge, 2000 MR:1798984(2001k:14041) 
[dF] de Fernex, T., Adjunction beyond thresholds and birationally rigid hypersurfaces; arxiv:math. AG/0604213

[dFEM] de Fernex, T, Ein, L., Mustaţă, M., Bounds on log-canonical thresholds with application to birational rigidity, Math. Res. Lett., 10, (2003), 219-236 MR1981899 (2004e:14060)

[DN] Drézét, J.-M., Narasimhan, M.S., Groupe de Picard des variétés de modules de fibrés semi-stables sur les courbes algébriques, Invent. Math., 97, (1989), 53-94 MR999313 (90d:14008)

[IM] Iskovskikh, V.A., Manin Ju. I., Three-dimensional quartics and counterexamples to the Luroth problem, Math. USSR-Sb., 15 (1971), 141-166

[K] Kollár, J.,Rational curves on algebraic varieties, Ergebnisse der Mathematik und ihrer Grenzgebiete. 3 Folge., 32, Springer-Verlag, Berlin, 1996 MR 1440180 (98c:14001)

[KMM] Kollár, J., Miyaoka, Y., Mori, S., Rationally Connected Varieties, J. Alg. Geom., I (1992), 429-448 MR1158625 (93i:14014)

[N] Newstead, P.E., Characteristic classes of stable bundles of rank 2 over an algebraic curve, Trans. Amer. Math. Soc., 169, (1972), 337-345 MR0316452 (47:4999)

[R] Ramanan, S., The moduli spaces of vector bundles over an algebraic curve, Math. Ann., 200, (1973), 69-84 MR0325615 (48:3962)

[P1] Pukhlikov, A.V., Birational automorphisms of Fano hypersurfaces, Invent. Math., 134, (1998), no. 2, 401-426 MR1650332(99i:14046)

[P2] Pukhlikov, A.V., Birationally rigid Fano double hypersurfaces, Sbornik: Mathematics, 191, (2000), no. 6, 101-126 MR1777571 (2001h:14054)

[P3] Pukhlikov, A.V., Birationally rigid Fano complete intersections, Crelle J. für die reine und angew. Math, 541, (2001), 55-79 MR1876285 (2003a:14015)

[P4] Pukhlikov, A.V., Birationally rigid Fano varieties, Proceedings of Fano Conference, 659681, Univ. Torino, Turin, 2004; arXiv:math.AG/0310267 MR2112597(2005j:14017)

Department of Mathematics, University of Texas at Austin, Austin, Texas 78712

E-mail address: noni@math.utexas.edu

Current address: Department of Mathematics, University of Massachusetts, Amherst, Massachusetts 01003

E-mail address: noni@math.umass.edu 